\title{
Identification of conserved microRNAs in peripheral blood from giant panda: expression of mammary gland-related microRNAs during late pregnancy and early lactation
}

\author{
C.D. Wang ${ }^{1,2 *}$, K. Long ${ }^{1 *}$, L. Jin ${ }^{1}$, S. Huang ${ }^{2}$, D.H. $\mathrm{Li}^{2}$, X.P. Ma ${ }^{3}$, M. Wei ${ }^{2}$, Y. Gu ${ }^{4}$, \\ J.D. $\mathrm{Ma}^{1}$ and $\mathrm{H}$. Zhang ${ }^{1,2}$ \\ ${ }^{1}$ College of Animal Science and Technology, Sichuan Agricultural University, Ya'an, \\ Sichuan, China \\ ${ }^{2}$ China Conservation and Research Center for the Giant Panda, Wolong, Sichuan, \\ China \\ ${ }^{3}$ Laboratory of Animal Disease and Human Health, Sichuan Agricultural University, \\ Ya'an, Sichuan, China \\ ${ }^{4}$ College of Life and Basic Sciences, Sichuan Agricultural University, Ya'an, China \\ *These authors contributed equally to this study. \\ Corresponding authors: J.D. Ma / H. Zhang \\ E-mail: jideng_ma@sina.com / wolong_zhm@126.com
}

Genet. Mol. Res. 14 (4): 14216-14228 (2015)

Received February 6, 2015

Accepted July 10, 2015

Published November 13, 2015

DOI http://dx.doi.org/10.4238/2015.November.13.5

\begin{abstract}
The giant panda (Ailuropoda melanoleuca) is one of the world's most endangered mammals, and it has evolved several unusual biological and behavioral traits. During puberty, pregnancy, lactation, and involution, the mammary gland undergoes profound morphological and functional changes. A large number of microRNAs (miRNAs) have been identified to be involved in mammary gland development and lactation. In this study, we identified 202 conserved mature miRNAs, corresponding to 147 pre-miRNAs, in giant panda peripheral blood using a small RNAsequencing approach. In addition, 27 miRNA families and 29 miRNA clusters
\end{abstract}


were identified. We analyzed the arm selection preference of pre-miRNAs and found that: 1) most giant panda pre-miRNAs generated one-strand miRNAs, and the $5 p$-arm only miRNAs have a higher expression level than $3 p$-arm only miRNAs; 2 ) there were more $5 p$-arm dominant miRNAs than $3 p$-arm dominant miRNAs; and 3) $5 p$-arm dominant miRNAs have a larger fold change within miRNA pairs than $3 p$-arm dominant miRNAs. Expression of 12 lactation-related miRNAs was detected across late pregnancy and early lactation stages by qPCR, and seven miRNAs were identified as clustered in one significant model. Most of these clustered miRNAs exhibited inhibitory roles in proliferation and differentiation of mammary epithelial cells. Functional analysis highlighted important roles of the seven as signed miRNAs in mammary development and metabolic changes, including blood vessel morphogenesis, macromolecule biosynthesis, cell cycle regulation, and protein transport.

Key words: Giant panda; miRNA; Mammary gland; Lactation

\section{INTRODUCTION}

The giant panda (Ailuropoda melanoleuca) is one of the world's most endangered mammals, and is distributed in a few mountain ranges in central China (Wei et al., 2015). The history of the giant panda goes back 7-8 million years during which it experienced two population expansions, two bottlenecks, and two divergences (Zhao et al., 2013). During its long history, the giant panda has evolved several unusual biological and behavioral traits, including a famously restricted diet primarily made up of bamboo, a very low fecundity rate, and anenlarged radial sesamoid that functions as a thumb (Zhao et al., 2010). The giant panda has not only the smallest neonate-maternal weight ratio (1:900) among the eutherian mammals, but also the longest gestation period (Kleiman, 1983). The low birth weight indicates that there is a large nutritional requirement at the early postnatal stage, which is provided by the maternal mammary gland.

MicroRNAs (miRNAs) are small regulatory RNAs composed of 20-30 nucleotides that are involved in the post-transcriptional regulation of gene expression. miRNAs play important roles in physiological and pathological processes such as development and cancer (Bartel, 2004). Nextgeneration sequencing technology and experimental studies have implicated many miRNAs in the regulation of mammary gland development. In 2009, Avril-Sassen et al. characterized miRNA expression in the mouse mammary gland over a 16-point developmental time course and found that miRNAs were expressed in co-regulated clusters. Li et al. (2012b) identified 56 significantly differentially expressed miRNAs between lactation and non-lactation periodsin goat using nextgeneration sequencing technology.

Difficulty obtaining tissue samples has impeded transcriptomic research of the mammary gland in giant panda. Blood can be described as a circulating 'connective' tissue, which connects the entire biological system at the physiological level. Because of this, blood profiling has been considered as a powerful means to investigate disease pathogenesis, physiological homeostasis, and the complexity of systems biology (Mohr and Liew, 2007). Blood cells express both organspecific genes and genes that are responsive to physiological stimuli; therefore, the blood transcriptome might be an accessible tool to investigate the multi-organ transcriptome (Kohane and Valtchinov, 2012). 
In this study, we performed small RNA sequencing of peripheral blood from the giant panda and, for the first time, identified 202 conserved mature miRNAs, corresponding to 147 premiRNAs, which belong to 27 miRNA families and 29 miRNA clusters. Analysis of chromosomal arm selection preference revealed that $5 p$-arm miRNAs are more highly expressed than $3 p$-arm miRNAs are. According to the expression of 12 lactation-related miRNAs across late gestation and early lactation stages, seven were assigned into a significant expression model using a short time-series expression miner (STEM) analysis. Target prediction and functional analysis showed that the seven assigned miRNAs have important roles in mammary development and metabolic changes, including a role in the vascular endothelial growth factor (VEGF) signaling pathway, blood vessel morphogenesis, negative regulation of macromolecule biosynthetic processes, the G1/S transition of the mitotic cell cycle, protein transport, and endoplasmic reticulum (ER) to Golgi vesicle-mediated transport.

\section{MATERIAL AND METHODS}

\section{Sample collection and RNA extraction}

Blood samples were collected from three giant pandas on days 78 (78 d) and 105 (105 d) of gestation, and days 3 ( 3 d), 20 (20 d), 56 (56 d), and 95 (95 d) of lactation. The blood sample used for small RNA sequencing was collected from a healthy female giant panda. All samples were stored at $-80^{\circ} \mathrm{C}$ for subsequent RNA extraction.

Total RNA was extracted from blood using TRIzol-LS (Invitrogen, Carlsbad, CA, USA), following the manufacturer protocol. RNA quality was analyzed with the Agilent Bioanalyzer 2100 and the RNA 6000 Nano LabChip Kit (Agilent, USA).

\section{Small RNA sequencing}

Total RNA isolated from three non-pregnant giant pandas was pooled in equal quantities. The constructed libraries were subjected to single-end sequencing in 36 nt reads using anlllumina Genome Analyzer II. The bioinformatics pipeline for miRNA discovery and profiling was carried out as previously described (Li et al., 2010a), with some modifications. All reads were counted and the identical reads were combined into a single kind.

There is a lack of giant panda miRNA information in miRBase. Therefore, to analyze conserved miRNAs, unique sRNAs were aligned with mammalian mature miRNAs in miRBase Release 20.0 (http://www.mirbase.org/). First, after rigorous screening, all retained sequences with ten or more copies were considered as potential miRNAs. Second, sRNA sequences with no more than four mismatched bases were selected by BLAST searching against miRBase. Third, the remaining 15-26 nt reads were used to map the giant panda genome and ESTs using the BLASTn program (Buhler et al., 2007). Sequences were retained for miRNA prediction. RNAfold (http://www.tbi.univie.ac.at/RNA/) was used for secondary structure prediction (hairpin prediction) of individual mappedmiRNAs, using the default folding conditions to identify conserved miRNAs.

\section{QuantitativePCR}

Expression of 12 selected miRNAs was quantified by aSYBRGreen-based High-Specificity miRNA qRT-PCR Detection Kit (Takara, Japan) on the CFX96 ${ }^{\text {TM }}$ Real-Time PCR Detection System 
(Bio-Rad, USA). We used U6 as the endogenous control gene. The $2^{-\Delta \Delta C t}$ method was employed to calculate the relative expression level samong diverse samples. All primer pairs are shown in Table1.

Table 1. Primer sequences used in the q-PCR experiments.

\begin{tabular}{lll}
\hline Gene & Primer & Primer sequence $\left(5^{\prime}-3^{\prime}\right)$ \\
\hline PANK3 & Forward primer & TTATGACAGATTTGGTCTGCC \\
& Reverse primer & AGAAAGCTTCAGTTGACCTT \\
TGF- $\beta$ & Forward primer & GAACTGGTGAAGCGGAAG \\
& Reverse primer & CCTCCTTGGCGTAGTAGT \\
PGR & Forward primer & GCTTCGTCCACTCAGGTA \\
IGF-1 & Reverse primer & CCTTCCTCTTCCTCCTTGA \\
& Forward primer & TCTGAGGAGGTTAGAGATGTA \\
TGF $\beta-R 1$ & Reverse primer & ACGATACTTCTGAGCCTTG \\
& Forward primer & AGATGGCAGAGTTGTGAAG \\
U6 & Reverse primer & CCTGTTGACTGAGTTGTGA \\
ame-miR-30b-5p & Forward primer & TTATGGGTCCTAGCCTGAC \\
ame-miR-15a-5p & Reverse primer & CACTATTGCGGGTCTGC \\
ame-miR-103a-1 & Forward primer & TGTAAACATCCTACACTCAGCT \\
ame-miR-205-5p & Forward primer & TAGCAGCACATAATGGTTTGT \\
ame-miR-93a & Forward primer & AGCAGCATTGTACAGGGCTATGA \\
ame-miR-221-3p & Forward primer & TCCTTCATTCCACCGGAGTCTGT \\
ame-miR-484-5p & ACTGCTGAGCCAGCACTTCCCGA \\
ame-let-7g & Forward primer & AGCTACATTGTCTGCTGGGTTT \\
ame-miR-200a & Forward primer & TCAGGCTCAGTCCCCTCCCGA \\
ame-let-7c & Forward primer & TGAGGTAGTAGTTTGTACAGTT \\
ame-miR-126-3p & Forward primer & TAACACTGTCTGGTAACGATGTT \\
ame-miR-129-5p & Forward primer & TGAGGTAGTAGGTTGTATGGTT \\
\hline
\end{tabular}

\section{STEM analysis}

STEM (Ernst and Bar-Joseph, 2006) is a software program that is used to cluster, compare, and visualize miRNA expression data from short time series experiments ( $\sim$ time points or fewer). The number of miRNAs expected to be assigned to a profile is estimated by random permutation. The statistical significance of the number of observed miRNAs assigned to each profile $(n)$ versus the number expected, $n(E)$, is also computed.

\section{Prediction and functional annotation of miRNA target genes}

PicTar (Krek et al., 2005) and TargetScan human 6.2 (Lewis et al., 2005) were used to predict the potential targets of a certain miRNA, and the overlaps of target genes from two programs were retained for further analysis. DAVID bioinformatic resource (Huang et al., 2008) was used to determine the potential biological function of predicted target genes. Gene ontology biological process (GO-BP) terms and KEGG pathway terms with $\mathrm{P}<0.01$ were chosen as potential functions.

\section{RESULTS}

\section{Discovery miRNAs in giant panda whole blood}

To comprehensively identify miRNAs in giant panda, we constructed a small RNA library from a giant panda blood sample. Deep sequencing of the small RNA library produced 7,063,822 raw sequence reads. After removing low quality sequences, adapters, sequences shorter than 16 
nt and known classes of RNAs (i.e., mRNA, rRNA, tRNA, snRNA, and snoRNA; and repetitive sequence elements), 6,898,133 "mappable reads" and 42,484 unique reads were retained for further analysis.

Through a set of rigorous criteria and sequence analysis, 202 conserved mature miRNAs and 147 pre-miRNAs were identified. Of the mature miRNAs, 186 were conserved in humans and other mammals, and 16 were conserved in non-human mammals. A feature of miRNAs is their size profile, which can distinguish them from other small RNAs. In the present study approximately half of the miRNAs identified were 22 nt long (46.53\%), followed by $21 \mathrm{nt}(24.75 \%)$ and $23 \mathrm{nt}(17.82)$, which is consistent with the typical size range for Dicer-derived products (Figure 1A).

The top 10, 20, and 50 miRNAs accounted for $76.99,89.96$, and $98.58 \%$ of the reads, respectively (Figure 1B). The top 10 miRNAs were ame-miR-486-5P, ame-miR-16-1-5P, amemiR-92a-3P, ame-miR-451a-5P, ame-miR-25-3P, ame-miR-93-5P, ame-let-7i-5P, ame-let-7b-5P, ame-miR-191-5P, and ame-let-7f-5P. ame-miR-486-5P was ranked first among all miRNAs having $1,143,323$ reads (Figure $1 \mathrm{C}$ ).
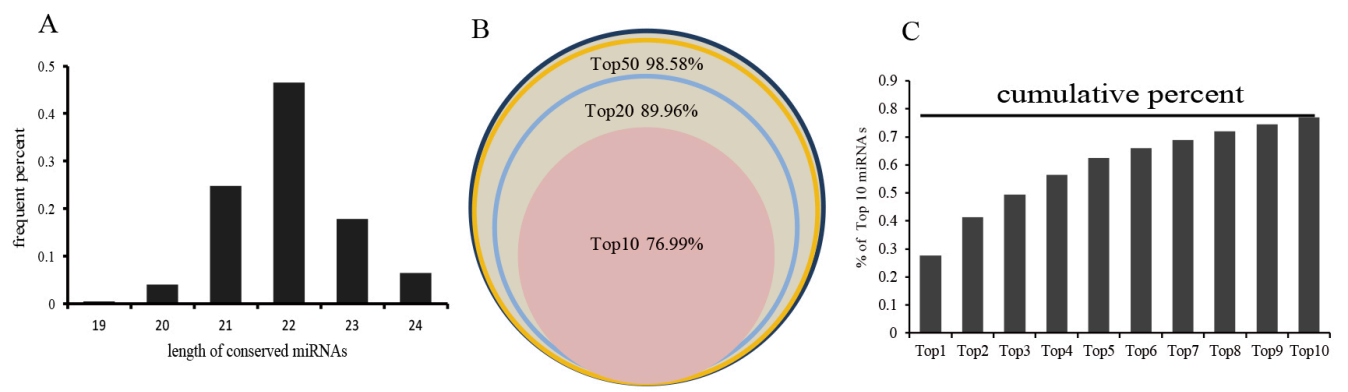

Figure 1. Length and count distribution of miRNAs in giant panda. A. Length distribution of miRNAs identified in whole blood from giant panda. B. Count distribution of thetop 10, 20, and 50 miRNAs. C. Plot of the top 10 miRNAs versus their accumulative percent in total counts of the mappable sequences.

\section{Arm selection preference of mature miRNAs}

Only one of the two strands generated from a precursor is preferentially processed into miRNA, whereasthe opposite arm (miR*) may quickly be degraded (Bartel, 2004). To investigate the arm selection preference of giant panda pre-miRNAs, the expression ratio of the $5 p$ arm to the $3 p$ arm was calculated. We divided pre-miRNAs into five groups, as previously described: $5 p$-arm only, 3p-arm only, 5p-arm dominant, 3p-arm dominant, and equal abundance (Li et al. 2010b). Most of the pre-miRNAs $(92,62.59 \%)$ were likely to generate single-stranded miRNAs $(5 p$-arm only or 3p-arm only), although a further 55 pre-miRNAs produced both strands ( $5 p$-arm dominant, $3 p$-arm dominant, or equal abundance) (Figure 2A). Interestingly, 5p-arm only pre-miRNAs had a higher average expression level than did 3p-arm only pre-miRNAs, with an approximately 3-fold difference in expression (Figure 2B).

A total of 49 pre-miRNAs showed at least a 2-fold difference in expression between the coupled miRNAs, of which 35 (23.81\%) had a higher 5p-arm expression level and $14(9.52 \%)$ had a relatively higher $3 p$-arm expression level (Figure $2 \mathrm{C}$ and $\mathrm{D}$ ). However, six pre-miRNAs showed no significant (fold change $<2$ ) expression difference (Figure 2A). In addition, we found the fold change in expression within miRNA pairs in 5p-arm dominant pre-miRNAs was higher than that in 3p-arm dominant pre-miRNAs (Figure 2C and 2D). All of five couple-expressed let-7 members 
have a higher $5 p$ expression level than $3 p$, For example, let-7b-5p expressed 122,754 reads and let-7b-3p expressed only 10 reads, with the highest fold change of 12,275 , which was consistent with previous reports from human studies (Hu et al. 2009). Collectively, we found that the 5 parm was expressed higher in pre-miRNAs in giant panda than the $3 p$ arm.
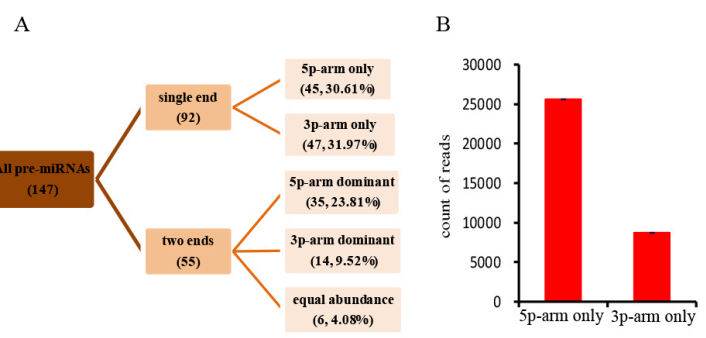
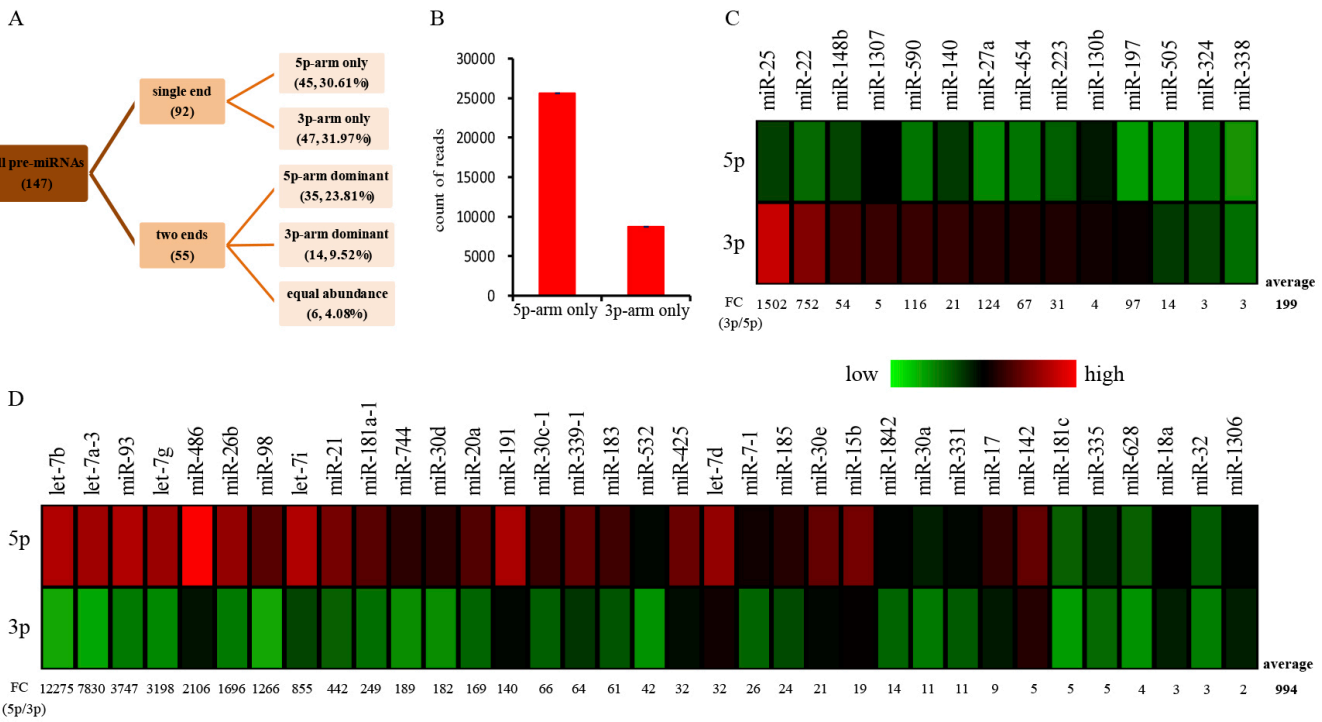

Figure 2. Arm selection preference of mature miRNAs isolated from giant panda. A. All pre-miRNAs were classified into five classes, $5 p$-arm only, 3p-arm only, $5 p$-arm dominant, $3 p$-arm dominant, and equal abundance. B. $5 p$-arm only miRNAs were more highly expressed than $3 p$-arm miRNAs were. C. D. Couple-expressed pre-miRNAs showed $3 p$ dominance (C) or $5 p$ dominance. (D). FC indicates the fold change between miRNA couples.

\section{miRNA families and clusters in giant panda}

miRNAs that are located close together within the genome have a similar pattern of expression and are co-regulated with host genes. According to the accepted inter-distance of 10 $\mathrm{kb}$ within one particular miRNA cluster, 29 clusters have been identified in giant panda (Table 2). These clusters have diverse numbers of members, ranging from two to eight. The largest cluster, which includes eight members, contains ame-miR-20a, ame-miR-92a-1, ame-miR-19b, ame-miR92a, ame-miR-17, ame-miR-19a, ame-miR-18a, and ame-miR-106a.

A miRNA family consists of a group of miRNAs that derive from a common ancestor, which regulate a similar set of target genes and therefore share similar biological and therapeutic function (Zou et al., 2014). Currently, the common criteria used for the classification of miRNA families are based on sequence similarity in the seed regions. We have identified 27 miRNA families that contain at least two members (Table 3). Two families contained six members, four had four members, five had three members, and 16 families had only two members. The wellknown let-7 family had six members, including ame-let-7c-5p, ame-let-7g-5p, ame-let-7i-5p, amelet-7b-5p, ame-let-7e-5p, and ame-miR-98-5p. The ame-miR-15 family also had six members, including ame-miR-15a-5p, ame-miR-15b-5p, ame-miR-195-5p, ame-miR-424-5p, ame-miR-497$5 p$, and ame-miR-322-5p. 
Table 2. miRNA clusters identified in giant panda.

\begin{tabular}{|c|c|c|c|c|c|}
\hline \multirow{2}{*}{$\frac{\text { Cluster ID }}{\text { Cluster } 1}$} & \multirow{2}{*}{$\frac{\text { No. of miRNAs }}{2}$} & \multicolumn{4}{|c|}{ Members of miRNAs } \\
\hline & & ame-miR-127 & ame-miR-136 & & \\
\hline Cluster 2 & 2 & ame-miR-133a & ame-miR-1 & & \\
\hline Cluster 3 & 2 & ame-miR-141 & ame-miR-200c & & \\
\hline Cluster 4 & 2 & ame-miR-145 & ame-miR-143 & & \\
\hline Cluster 5 & 2 & ame-miR-181c & ame-miR-181d & & \\
\hline Cluster 6 & 2 & ame-miR-191 & ame-miR-425 & & \\
\hline Cluster 7 & 2 & ame-miR-195 & ame-miR-497 & & \\
\hline Cluster 8 & 2 & ame-miR-221 & ame-miR-222 & & \\
\hline Cluster 9 & 2 & ame-miR-27b & ame-miR-23b & & \\
\hline Cluster 10 & 2 & ame-miR-301b & ame-miR-130b & & \\
\hline Cluster 11 & 2 & ame-miR-30d & ame-miR-30b & & \\
\hline Cluster 12 & 2 & ame-miR-34b & ame-miR-34c & & \\
\hline Cluster 13 & 2 & ame-miR-98 & ame-let-7f & & \\
\hline Cluster 14 & 2 & ame-miR-99a & ame-let-7c & & \\
\hline Cluster 15 & 3 & ame-miR-181b & ame-miR-181a-2 & ame-miR-181a & \\
\hline Cluster 16 & 3 & ame-miR-200b & ame-miR-200a & ame-miR-429 & \\
\hline Cluster 18 & 3 & ame-miR-25 & ame-miR-106b & ame-miR-93 & \\
\hline Cluster 19 & 3 & ame-miR-30e & ame-miR-30c-1 & ame-miR-30c & \\
\hline Cluster 20 & 3 & ame-miR-450a & ame-miR-542 & ame-miR-424 & \\
\hline Cluster 21 & 3 & ame-miR-96 & ame-miR-183 & ame-miR-182 & \\
\hline Cluster 22 & 3 & ame-let-7a & ame-let-7d & ame-let-7f-1 & \\
\hline Cluster 23 & 3 & ame-let-7e & ame-miR-125a & ame-miR-99b & \\
\hline Cluster 24 & 4 & ame-miR-15b & ame-miR-16 & ame-miR-16-2 & ame-miR-15a \\
\hline Cluster 25 & 3 & ame-miR-23a & ame-miR-24-2 & ame-miR-24 & \\
\hline Cluster 26 & 4 & ame-miR-29b & ame-miR-29c & ame-miR-29b-2 & ame-miR-29a \\
\hline Cluster 27 & 4 & ame-miR-381 & ame-miR-409 & ame-miR-379 & ame-miR-411 \\
\hline \multirow[t]{2}{*}{ Cluster 28} & 7 & ame-miR-362 & ame-miR-500a & ame-miR-500b & ame-miR-502 \\
\hline & & ame-miR-532 & ame-miR-660 & ame-miR-500 & \\
\hline \multirow[t]{2}{*}{ Cluster 29} & 8 & ame-miR-20a & ame-miR-92a-1 & ame-miR-19b & ame-miR-92a \\
\hline & & ame-miR-17 & ame-miR-19a & ame-miR-18a & ame-miR-106a \\
\hline
\end{tabular}

\section{miRNAs related to mammary development had asimilar expression pattern}

To investigate the expression pattern of lactation-related miRNAs in giant panda mammary development during transition periods, we studied the expression level of 12 mammary gland development-related miRNAs by quantitative (q)-PCR across the second half of pregnancy and over multiple lactation stages. To illustrate the expression pattern of these miRNAs at various stages of mammary development, STEM analysis was performed. This revealed that seven of 12 miRNAs were assigned into one significant expression model $(P<0.01)$, which showed that the lowest level of expression occurred post-parturition, following a dramatic increase on lactation $3 \mathrm{~d}$, and then a gradual decrease from lactation $3 \mathrm{~d}$ to lactation $20 \mathrm{~d}$, and was ultimately steady during the later lactation stages (Figure 3). The dynamic expression of these seven miRNAs suggests that they have important roles in mammary gland development and lactation.

All seven of these miRNAs are involved in mammary development, and metabolic and nutrient secretion activities of mammary epithelial cells. Evidence has shown that overexpression of miR-103-1 increases fat droplet formation, triglyceride accumulation, and the proportion of unsaturated fatty acids (Lin et al., 2013). High expression of miR-103-1 at lactation $3 \mathrm{~d}$ might be involved in the initiation of lipid and fat secretion during early lactation. Furthermore, miR-126-3P, miR-129-5P, miR-484-5p, miR-93a, and miR-30b are important in the inhibition of mammary cell proliferation or differentiation (Cui et al., 2011; Li et al., 2012a; Le Guillou et al., 2012). For example, $\mathrm{miR}-126-3 p$ inhibited protein expression of progesterone receptor (PGR) and the proliferation of mammary epithelial cells, and therefore played an important role in the process of mammary gland 
development (Cui et al., 2011).The miRNA-30b family might play a role in the differentiation of adipocytes (Lin et al., 2010; Zaragosi et al., 2011), B-cells (Lin et al., 2010), and osteoblasts (Wu et al., 2012). Transgenic mice overexpressing miR-30b in mammary epithelial cells showed a reduction in the size of alveolar lumen and down-expression of the ErbB family members: ErbB2, which is involved in the formation of mammary secretory alveoli, and ErbB4, which inhibits the growth of mammary epithelial cells (Muraoka-Cook et al., 2008).

\begin{tabular}{|c|c|c|c|c|c|}
\hline miRNA family & Seed sequence & Number of unique members & & Family members & \\
\hline 1 & GAGGTAG & 6 & $\begin{array}{l}\text { ame-miR-98-5p } \\
\text { ame-let-7i-5p }\end{array}$ & $\begin{array}{l}\text { ame-let-7c-5p } \\
\text { ame-let-7b-5p }\end{array}$ & $\begin{array}{l}\text { ame-let-7g-5p } \\
\text { ame-let-7e-5p }\end{array}$ \\
\hline 2 & AGCAGCA & 6 & $\begin{array}{l}\text { ame-miR-15a-5p } \\
\text { ame-miR-424-5p }\end{array}$ & $\begin{array}{l}\text { ame-miR-15b-5p } \\
\text { ame-miR-497-5p }\end{array}$ & $\begin{array}{l}\text { ame-miR-195-5p } \\
\text { ame-miR-322-5p }\end{array}$ \\
\hline 3 & AAAGTGC & 4 & $\begin{array}{l}\text { ame-miR-20b-5p } \\
\text { ame-miR-106b-5p }\end{array}$ & ame-miR-20a-5p & ame-miR-93-5p \\
\hline 4 & ACATTCA & 4 & $\begin{array}{l}\text { ame-miR-181a-5p } \\
\text { ame-miR-181d-5p }\end{array}$ & ame-miR-181b-5p & ame-miR-181c-5p \\
\hline 5 & GTAAACA & 4 & $\begin{array}{l}\text { ame-miR-30d-5p } \\
\text { ame-miR-30a-5p }\end{array}$ & ame-miR-30b-5p & ame-miR-30e-5p \\
\hline 6 & AGTGCAA & 4 & ame-miR-130a-3p & ame-miR-130b-3p & ame-miR-301a-3p \\
\hline 7 & CAGTGCA & 3 & ame-miR-148a-3p & ame-miR-148b-3p & ame-miR-152-3p \\
\hline 8 & AGCACCA & 3 & ame-miR-29a-3p & ame-miR-29c-3p & ame-miR-21-3p \\
\hline 9 & GGCAGTG & 3 & ame-miR-34a-5p & ame-miR-34c-5p & ame-miR-34b-5p \\
\hline 10 & ATTGCAC & 3 & ame-miR-25-3p & ame-miR-363-3p & \\
\hline 11 & AATACTG & 3 & ame-miR-200c-3p & ame-miR-200b-3p & ame-miR-429-3p \\
\hline 12 & TATACAA & 2 & ame-miR-98-3p & ame-let-7f-1-3p & \\
\hline 13 & GAGAACT & 2 & ame-miR-146a-5p & ame-miR-146b-5p & \\
\hline 14 & AAGTTCT & 2 & ame-miR-148a-5p & ame-miR-148b-5p & \\
\hline 15 & CCATCGA & 2 & ame-miR-181c-3p & ame-miR-181a-2-3p & \\
\hline 16 & AAGGTGC & 2 & ame-miR-18b-5p & ame-miR-18a-5p & \\
\hline 17 & ACTGGCC & 2 & ame-miR-193a-3p & ame-miR-193b-3p & \\
\hline 18 & GTGCAAA & 2 & ame-miR-19a-3p & ame-miR-19b-3p & \\
\hline 19 & AACACTG & 2 & ame-miR-141-3p & ame-miR-200a-3p & \\
\hline 20 & ATCTTAC & 2 & ame-miR-200b-5p & ame-miR-200a-5p & \\
\hline 21 & GCTACAT & 2 & ame-miR-221-3p & ame-miR-222-3p & \\
\hline 22 & TCACATT & 2 & ame-miR-23b-3p & ame-miR-23a-3p & \\
\hline 23 & TTCAGTC & 2 & ame-miR-30d-3p & ame-miR-30a-3p & \\
\hline 24 & TTTCAGT & 2 & ame-miR-30e-3p & ame-miR-30a-3p & \\
\hline 25 & ATCACTA & 2 & ame-miR-34c-3p & ame-miR-34b-3p & \\
\hline 26 & AAGCTCG & 2 & ame-miR-99a-3p & ame-miR-99b-3p & \\
\hline 27 & АCССTGT & 2 & ame-miR-10a-5p & ame-miR-10b-5p & \\
\hline
\end{tabular}

Consistent with previous studies, we also determined the expression level of five experimentally validated target mRNAs; transforming growth factor-beta (TGF- $\beta / \beta 1), P G R$, insulinlike growth factor 1 , and pantothenate kinase 3 (PANK3) by qPCR (Figure 4). miR-103-1 is located in the sense oriented intron 5 of PANK3. PANK enzymes are thought to be important metabolic regulators due to their capacity of catalyzing the rate-limiting step in coenzyme A synthesis. Although it does not directly target the 3'UTR of PANK3, miR-103-1 can positively regulate the transcription of PANK3 indirectly (Lin et al., 2013). In this study, we observed a strong positive correlation between miR-103-1 and PANK3 $(r=0.85, \mathrm{P}<0.001)$, suggesting that miR-103-1 affects the metabolic process. In addition, four mRNAs showed a significant negative correlation with their corresponding miRNAs, with the correlation index ranging from -0.81 to -0.84 . PGR mediates the action of progesterone, which is essential for mammary epithelial proliferation and differentiation during pregnancy (Topper and Freeman, 1980). Whilst it was reported that miR-126-5p did not down-regulate protein expression of PGR, the same was not found for mRNA. The lowest level 
of PGR expression, which corresponded to the peak in miR-126-5p expression at lactation $3 \mathrm{~d}$, indicates a decreased proliferation rate of mammary epithelial cells (Cui et al., 2011).In dairy cows, the onset of lactation is characterized by a decreased rate of cell differentiation and proliferation corresponding to decreased gene expression in these and related processes (Finucane et al., 2008). Taken together, it is reasonable to speculate that the rate of cell proliferation and differentiation of giant panda mammary gland cells may decrease during early lactation, and this is supported by the peak expression of some growth-inhibitory miRNAs at lactation $3 \mathrm{~d}$.

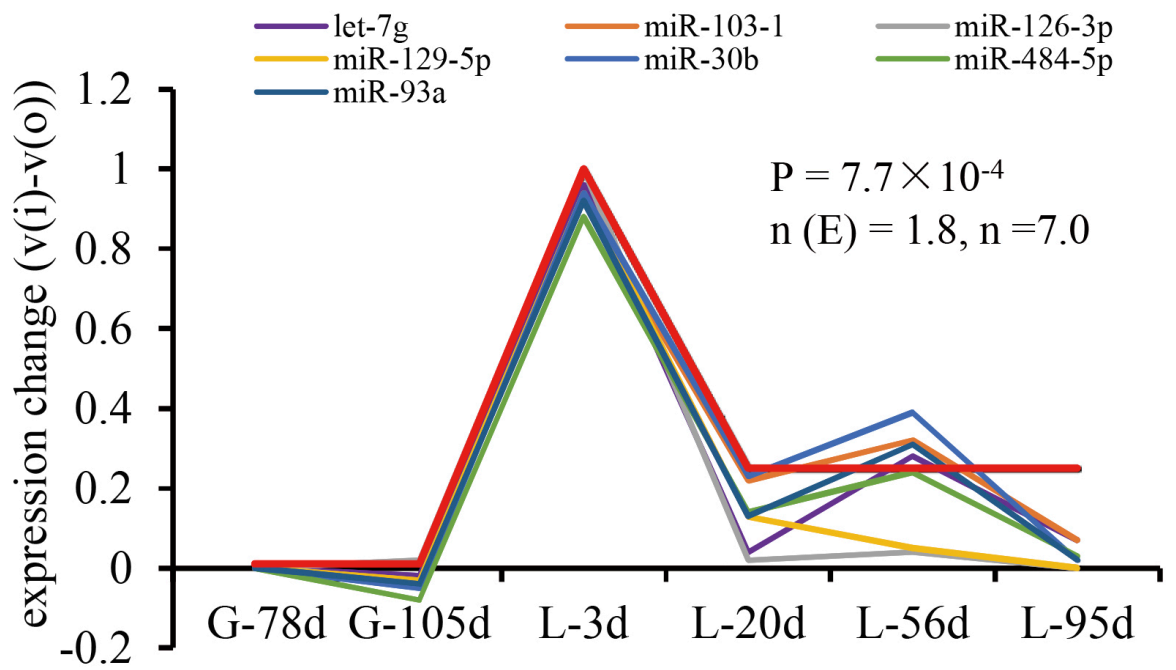

Figure 3. Expression pattern of significantly clustered miRNAs. Seven of 12 miRNAs showed a significant clustering pattern. G-78 d, G-105 d indicated gestation 78 d and gestation 105 d, respectively. L-3 d, L-20 d, L-56 d, L-95 d present lactation $3 \mathrm{~d}, 20 \mathrm{~d}, 56 \mathrm{~d}, 95 \mathrm{~d}$, respectively. $n(E)$ and $n$ indicate the expected miRNA number and the observed miRNA number assigned into this cluster, respectively.
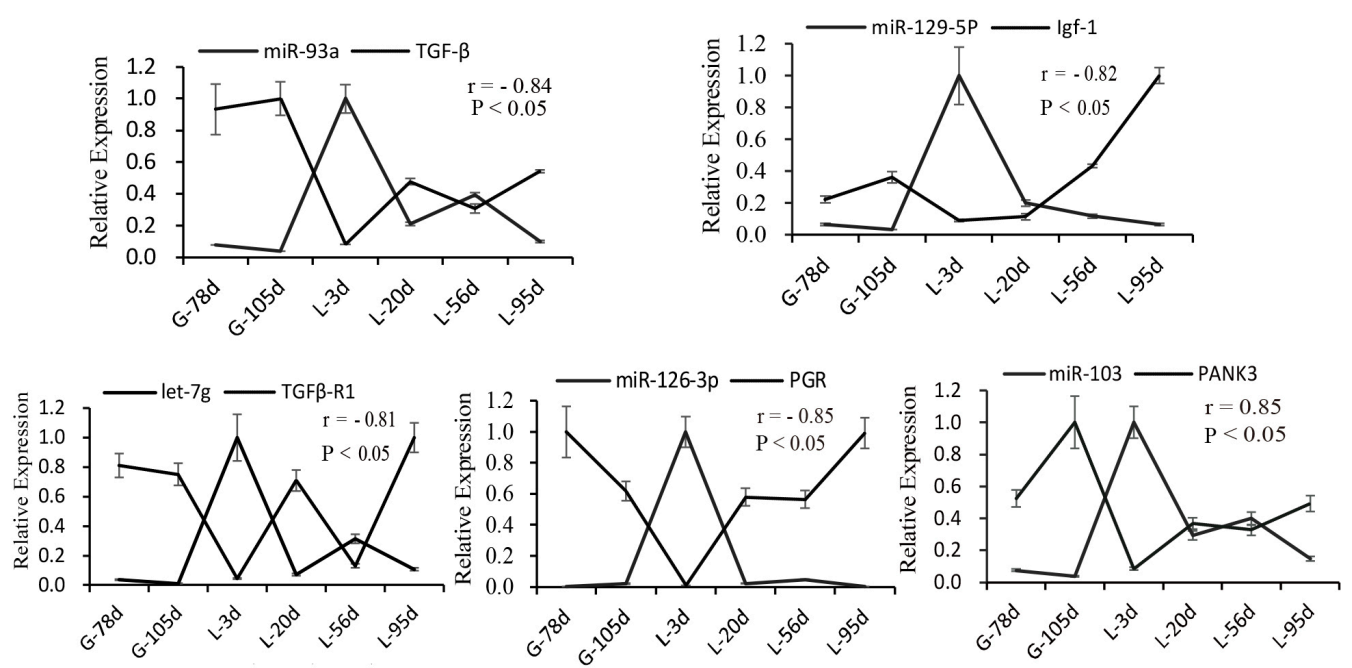

Figure 4. Pearson's correlation analysis between miRNA and corresponding target mRNA. 


\section{Functional analysis of the significant clustering miRNAs}

Given that similar expression pattern might indicate similar physiological functions, we predicted the target genes and performed GO analysis to further investigate the potential roles of these seven identified miRNAs. The Online target prediction software (TargetScan and Pictar) was used to predict target genes, and 966 target genes that appeared in both programs were retained for $\mathrm{GO}$ analysis.

As shown in Figure 5, significant clusters of miRNAs were involved in the VEGF signaling pathway, blood vessel morphogenesis, negative regulation of macromolecule biosynthetic processes, the G1/S transition of the mitotic cell cycle, protein transport, and ER to Golgi vesiclemediated transport. These biological processes play important roles in mammary gland development and activity performance. VEGF signaling is involved in a series of different pathways, which ultimately mediate the proliferation and migration of endothelial cells, enhancing their vascular permeability to water and proteins. Protein transport is the directed movement of proteins into, out of, and within a cell, or between cells, by means of some agent such as a transporter or pore. ER to Golgi vesicle-mediated transport is the directed movement of substances from the ER to the Golgi. Microtubule-based transport is the directed movement of organelles or other particles from one location in the cell to another. All of these three processes are necessary for the secretion of proteins into milk. In addition, the G1/S transition of the mitotic cell cycle is the point in which a cell in $\mathrm{G} 1$ commits to entering the $\mathrm{S}$ phase, which is an important step in proliferation.

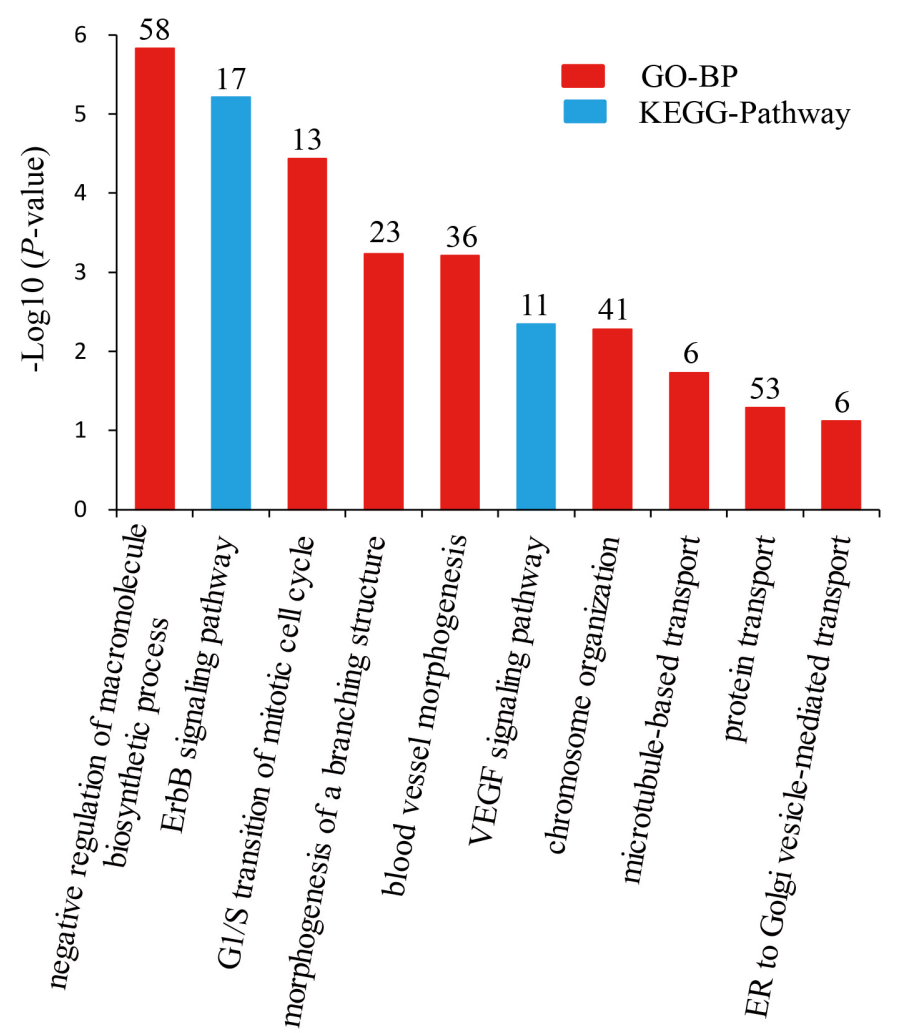

Figure 5. Gene Ontology and KEGG pathway terms with the highest significance of functional clusters. 


\section{DISCUSSION}

Next-generation sequencing technologies have been used to identify miRNAs in diverse species. To date, 24,521 miRNA loci from 206 species, expressing 30,424 mature miRNAs have been deposited in the public miRNA database, miRbase (released 20, July 2013). However, few studies have reported miRNAs in giant panda, which is of interest to biologists because of the unusual biological traits of this species. To our knowledge, this is the first comprehensive identification of miRNAs in giant panda. In this study, a blood sample was used to generate a small RNA library containing 7,000,600 raw reads and 6,900,000 clean reads. A total of 202 mature miRNAs and 147 pre-miRNAs that were conserved in mammals were identified in the giant panda.

We analyzed the arm selection preference of pre-miRNAs and found that: 1) most of the pre-miRNAs generated one-strand miRNA, and 5p-arm only miRNAs are more highly expressed than 3p-arm only miRNAs; 2 ) there were more $5 p$-arm dominant miRNAs than 3p-arm dominant miRNAs; and 3) 5p-arm dominant miRNAs have a larger fold change within miRNA pairs than $3 p$-arm dominant miRNAs. Collectively, 5p-arm miRNAs were more highly expressed than 3p-arm miRNAs were. This trend, which results from Drosha enzymes having higher accuracy than Dicer enzymes, is distinctively different among various species. In humans, Hu et al. (2009) found highly expressed miRNAs to be biased towards the $5 p$ hairpin arm, but no similar observation was made in flies. In medakas, no significant bias was observed between $5 p$-dominant and $3 p$-dominant miRNAs, with $6.7 \%$ and $5.2 \%$ accounting for $5 p$-dominant and $3 p$-dominant miRNAs, respectively (Li et al., 2010b). In addition, we identified 27 families and 29 clusters in giant panda, the majority of which were conserved with other species.

In this study, we revealed that seven miRNAs (let-7g, miR-103-1, miR-126-3p, miR-129-5p, miR-30b, miR-484-5p, andmiR-93a) have a similar expression pattern, which involves the lowest expression in late gestation, which then peaks in the early lactation period, and remains stable in the later stages of lactation. Many significant clustering miRNAs play important roles in inhibiting cell differentiation and proliferation. During lactogenesis, the mammary gland undergoes dramatic functional and metabolic changes during the transition from late pregnancy to lactation. The secretory capacity of the mammary gland is determined by the number of mammary epithelial cells and their secretory activity. To support high levels of milk production, the enzymatic and transport activities of the mammary gland increase significantly from the non-lactating to the lactation period. The rapid increase of enzymatic and transport activities may result from an increase in the number of epithelial cells or increased cellular activity (Capuco et al., 2003).

The population of secretory cells increases dramatically during pregnancy; in some species the story ends there, but not in all. In the Guinea pig, there is relatively little change in DNA $_{t}$ levels during gestation, but a large increase occurs within 2 days of parturition. It is well established that a transient surge of cell proliferation occurs 2 or 3 days post-partum in mouse and rat. However, the DNA concentration remains relatively unchanged during early lactation in some large mammalian species such as sheep (Anderson, 1975), goat (Anderson et al., 1981), and cow (Baldwin, 1966). In 2008, Finucane et al. (2008) analyzed expression profiles of bovine mammary tissue about 5 days before parturition and on day 10 after parturition. The result showed that up-regulated genes were associated with transport, lipid and carbohydrate metabolism, and cell signaling, whereas down-regulated genes were associated with cell cycle and proliferation, DNA replication and chromosome organization, and microtubule-based processes. Characteristics of mammary glands during the transition period in giant panda have not been described. In this study, we found some miRNAs that inhibit cell proliferation and differentiation to be more abundance in 
the early lactation period, which is consistent with the observation in cows. Taken together, we suggest that the functional and morphological changes that occur in the mammary epithelial cells of giant panda are similar to those that occur in cows, including a transient decrease or pause in proliferation, and a dramatic increase in metabolism during the early stage of lactation.

In this study, we sequenced small RNAs in the peripheral blood of a giant panda and, for the first time, identified 202 conserved mature miRNAs, corresponding to 147 pre-miRNAs belonging to 27 miRNA families and 29 miRNA clusters. Analysis of arm selection preference revealed that $5 p$-arm only, 3p-arm only, 5p-arm dominant, 3p-arm dominant, and equal abundance miRNAs account for $30.61,31.97,23.81,9.52$, and $4.08 \%$ of the total miRNA abundance, respectively. To investigate the pattern of lactation-related miRNA expression during mammary gland development, we determined the level of expression of 12 lactation-related miRNAs by qPCR across late gestation and early lactation stages. The results showed that seven of these 12 miRNAs were assigned into a significant expression model under STEM analysis. We also determined the expression level of five experimentally validated target genes and revealed a significant correlation between miRNAs and the corresponding mRNA. Target prediction and functional analysis highlighted important roles for the seven assigned miRNAs in mammary gland development and metabolism, including involvement in the VEGF signaling pathway, blood vessel morphogenesis, negative regulation of macromolecule biosynthetic processes, G1/S transition of the mitotic cell cycle, protein transport, and ER to Golgi vesicle-mediated transport.

\section{Conflicts of interest}

The authors declare no conflicts of interest.

\section{ACKNOWLEDGMENTS}

Research supported by grants from Giant Panda International Projects (\#AD1416).

\section{REFERENCES}

Anderson R (1975). Mammary gland growth in sheep. J. Anim. Sci. 41: 118-123.

Anderson R, Harness J, Snead A and Salah M (1981). Mammary growth pattern in goats during pregnancy and lactation. J. Dairy Sci. 64: 427-432.

Avril-Sassen S, Goldstein LD, Stingl J, Blenkiron C, et al. (2009). Characterisation of microRNA expression in post-natal mouse mammary gland development. BMC Genomics. 10: 548.

Baldwin R (1966). Enzymatic activities in mammary glands of several species. J. Dairy Sci. 49:1533-1542.

Bartel DP (2004). MicroRNAs: genomics, biogenesis, mechanism, and function. Cell 116: 281-297.

Buhler JD, Lancaster JM, Jacob AC and Chamberlain RD (2007). Mercury BLASTN: Faster DNA sequence comparison using a streaming hardware architecture. Proc. of Reconfigurable Systems Summer Institute.

Capuco AV, Ellis SE, Hale SA, Long E, et al. (2003). Lactation persistency: insights from mammary cell proliferation studies. J. Anim. Sci. 81: 18-31.

Cui W, Li Q, Feng L and Ding W (2011). MiR-126-3p regulates progesterone receptors and involves development and lactation of mouse mammary gland. Mol. Cell Biochem. 355: 17-25.

Ernst $\mathrm{J}$ and Bar-Joseph Z (2006). STEM: a tool for the analysis of short time series gene expression data. BMC Bioinformatics 7: 191.

Finucane KA, McFadden TB, Bond JP, Kennelly JJ, et al. (2008). Onset of lactation in the bovine mammary gland: gene expression profiling indicates a strong inhibition of gene expression in cell proliferation. Funct. Integrat. Genomics 8: 251-264.

$\mathrm{Hu} \mathrm{HY}$, Yan Z, Xu Y, Hu H, et al. (2009). Sequence features associated with microRNA strand selection in humans and flies. BMC Genomics 10: 413. 
Huang DW, Sherman BT and Lempicki RA (2008). Systematic and integrative analysis of large gene lists using DAVID bioinformatics resources. Nat. Protoc. 4: 44-57.

Kleiman DG (1983). Ethology and Reproduction of Captive Giant Pandas (Ailuropoda melanoleuca). Zeitschrift für Tierpsychologie. 62: 1-46.

Kohane IS and Valtchinov VI (2012). Quantifying the white blood cell transcriptome as an accessible window to the multiorgan transcriptome. Bioinformatics 28: 538-545.

Krek A, Grün D, Poy MN, Wolf R, et al. (2005). Combinatorial microRNA target predictions. Nat. Genet. 37: 495-500.

Le Guillou S, Sdassi N, Laubier J, Passet B, et al. (2012). Overexpression of miR-30b in the developing mouse mammary gland causes a lactation defect and delays involution. PLoS One 7: e45727.

Lewis BP, Burge CB and Bartel DP (2005). Conserved seed pairing, often flanked by adenosines, indicates that thousands of human genes are microRNA targets. Cell 120: 15-20.

Li HM, Wang CM, Li QZ and Gao XJ (2012a). MiR-15a decreases bovine mammary epithelial cell viability and lactation and regulates growth hormone receptor expression. Molecules 17: 12037-12048.

Li M, Xia Y, Gu Y, Zhang K, et al. (2010a). MicroRNAome of porcine pre-and postnatal development. PLoS One 5:e11541.

Li SC, Chan WC, Ho MR, Tsai KW, et al. (2010b). Discovery and characterization of medaka miRNA genes by next generation sequencing platform. BMC Genomics 11: S8.

Li Z, Liu H, Jin X, Lo L, et al. (2012b). Expression profiles of microRNAs from lactating and non-lactating bovine mammary glands and identification of miRNA related to lactation. BMC Genomics 13: 731.

Lin J, Lwin T, Zhao J, Tam W, et al. (2010). Follicular dendritic cell-induced microRNA-mediated upregulation of PRDM1 and downregulation of BCL-6 in non-Hodgkin's B-cell lymphomas. Leukemia 25: 145-152.

Lin X, Luo J, Zhang L, Wang W, et al. (2013). MiR-103 Controls Milk fat accumulation in goat (Capra hircus) mammary gland during lactation. PLoS One 8: e79258.

Mohr S and Liew CC (2007). The peripheral-blood transcriptome: new insights into disease and risk assessment. Trends Mol. Med. 13: 422-432.

Muraoka-Cook RS, Feng SM, Strunk KE and Earp HS (2008). ErbB4/HER4: Role in Mammary Gland Development, Differentiation and Growth Inhibition. J. Mammary Gland Biol. 13: 235-246.

Topper YJ and Freeman CS (1980). Multiple hormone interactions in the developmental biology of the mammary gland. Physiol. Rev. 60: 1049-1106.

Wei F, Hu Y, Yan L, Nie Y, et al. (2015). Giant Pandas are not an evolutionary cul-de-sac: Evidence from multidisciplinary research. Mol. Biol. Evol. 32: 4-12.

Wu T, Zhou H, Hong Y, Li J, et al. (2012) miR-30 family members negatively regulate osteoblast differentiation. J. Biol. Chem. 287: 7503-7511.

Zaragosi LE, Wdziekonski B, Brigand KL, Villageois P, et al. (2011). Small RNA sequencing reveals miR-642a-3p as a novel adipocyte-specific microRNA and miR-30 as a key regulator of human adipogenesis. Genome Biol. 12: R64.

Zhao H, Yang JR, Xu Hand Zhang J (2010). Pseudogenization of the umami taste receptor gene Tas1r1 in the giant panda coincided with its dietary switch to bamboo. Mol. Biol. Evol. 27: 2669-2673.

Zhao S, Zheng P, Dong S, Zhan X, et al. (2013). Whole-genome sequencing of giant pandas provides insights into demographic history and local adaptation. Nat. Genet. 45: 67-71.

Zou Q, Mao Y, Hu L, Wu Y, et al. (2014). miRClassify: An advanced web server for miRNA family classification and annotation. Comput. Biol. Med. 45: 157-160. 